\title{
Excess information in Parametric Linear
}

\section{Optimization*}

\author{
M.A. GOBERNA ${ }^{a, \dagger}$, V. JORNET $^{a}$ and M. MOLINA ${ }^{a}$ \\ ${ }^{a}$ Department of Statistics and Operations Researchy, Faculty of Sciences, University of Alicante, San \\ Vicente del Raspeig, 03080 Alicante, Spain.
}

\begin{abstract}
We consider a parametric linear optimization problem (called primal) and its corresponding dual problem, where the parameters are the cost vector and the right-hand-side vector, respectively. This paper characterizes those constraints of the primal problem (variables of the dual problem, respectively) which can be eliminated without modifying its feasible set mapping, its optimal set mapping, and its value mapping. Superfluity relative to the primal feasible set is nothing else than redundancy in its constraint system, whereas superfluity relative to the dual optimal set is closely related with another well-known phenomenon of excess of information in linear optimization: strong strangeness. The relationships between all these phenomena are also analyzed.
\end{abstract}

Key Words: linear inequality systems, linear programming, linear semi-infinite programming, excess of information, redundancy.

Mathematics Subject Classifications 2000: 90C34; 90C05

\section{Introduction}

We consider given a linear system in $\mathbb{R}^{n}, \sigma=\left\{a_{t}^{\prime} x \geq b_{t}, t \in T\right\}$, where $T$ is an arbitrary index set with cardinality $2 \leq|T| \leq \infty$. We associate with $\sigma$ the parametric linear optimization problem

$0 *$ This work was supported by the DGES of Spain, Grant PB98-0975.

${ }^{\dagger}$ Corresponding author. E-mail: mgoberna@ua.es 


$$
P(c): \quad \operatorname{Inf} c^{\prime} x \quad \text { s.t. } \quad a_{t}^{\prime} x \geq b_{t}, t \in T
$$

and its dual

$$
D(c): \quad \operatorname{Sup} \Psi(\lambda):=\sum_{t \in T} \lambda_{t} b_{t} \quad \text { s.t. } \quad \sum_{t \in T} \lambda_{t} a_{t}=c, \lambda \in \mathbb{R}_{+}^{(T)}
$$

where the parameter is $c \in \mathbb{R}^{n}$ whereas $X^{(T)}$, with $0 \in X \subset \mathbb{R}$, denotes the set of the mappings $\lambda: T \rightarrow X$ vanishing everywhere except on a finite subset of $T$, $\operatorname{supp} \lambda=\left\{t \in T \mid \lambda_{t} \neq 0\right\}$. We denote by $F, F^{*}(c)$ and $v(c)$ the feasible set, the optimal set and the optimal value of $P(c)$, and we denote by $\Lambda(c), \Lambda^{*}(c)$ and $v^{D}(c)$ the feasible set, the optimal set and the optimal value of $D(c)$, respectively. By definition, $v(c)=+\infty$ if $F=\emptyset$ and $v^{D}(c)=-\infty$ if $\Lambda(c)=\emptyset$.

If $|T|<\infty, P(c)$ is a linear programming (LP) problem in canonical form and $D(c)$ is a LP problem in standard form. In this case, if at least one of the two problems is bounded, then they are solvable and $v(c)=v^{D}(c)$.

If $|T|=\infty, P(c)$ is a primal linear semi-infinite (LSIP) problem and $D(c)$ is its Haar's dual problem. It is possible that $v^{D}(c)<v(c)$ even though both problems (possibly unsolvable) are consistent.

We consider the feasible set of $P(c), F$ (which can be seen as a constant set-valued mapping), the set-valued mappings $F^{*}: \mathbb{R}^{n} \rightrightarrows \mathbb{R}^{n}$ and $\Lambda, \Lambda^{*}: \mathbb{R}^{n} \rightrightarrows \mathbb{R}^{(T)}$, and the ordinary mappings $v, v^{D}: \mathbb{R}^{n} \rightarrow \overline{\mathbb{R}}$. Similarly, given an index $s \in T$, we associate with the relaxed system $\sigma_{s}:=\left\{a_{t}^{\prime} x \geq b_{t}, t \in T \backslash\{s\}\right\}$ its corresponding parametric problems, $P_{s}(c)$ and $D_{s}(c)$, and their corresponding mappings: $F_{s}, F_{s}^{*}, \Lambda_{s}, \Lambda_{s}^{*}, v_{s}$ and $v_{s}^{D}$.

We say that the constraint $a_{s}^{\prime} x \geq b_{s}$ (the variable $\lambda_{s}$ ) is superfluous relative to one of the mappings associated with $P(c)(D(c)$, respectively) if its elimination does not modify the corresponding mapping. For the sake of brevity we say that $s \in T$ is superfluous in both cases. In particular, $s$ is superfluous relative to the primal feasible set if $F_{s}=F$ (i.e., $s$ is redundant), $s$ is superfluous relative to the primal value function (PVS) if $v_{s}=v$, and $s$ is superfluous relative to the primal optimal set (POS) if $F_{s}^{*}=F^{*}$. Similarly, we say that $s$ is superfluous relative to the dual optimal value (DVS) if $v_{s}^{D}=v^{D}$. 
Concerning the dual set-valued mappings, since the decision spaces of $D(c)$ and $D_{s}(c)$ are $\mathbb{R}^{(T)}$ and $\mathbb{R}^{(T \backslash\{s\})}$, respectively, the comparison of subsets requires the identification of each subset of $\mathbb{R}^{(T \backslash\{s\})}$ with another one in $\mathbb{R}^{(T)}$. The natural way to do this consists of associating with each $\gamma \in \mathbb{R}^{(T \backslash\{s\})}$ its extension with zero $\bar{\gamma} \in \mathbb{R}^{(T)}$, i.e.,

$$
\bar{\gamma}_{t}= \begin{cases}\gamma_{t}, & t \in T \backslash\{s\} \\ 0, & t=s\end{cases}
$$

so that we identify any set $\Gamma \subset \mathbb{R}^{(T \backslash\{s\})}$ with the set $\bar{\Gamma}:=\left\{\bar{\gamma} \in \mathbb{R}^{(T)} \mid \gamma \in \Gamma\right\}$. In particular, we have $\overline{\Lambda_{s}(c)} \subset \Lambda(c)$ for all $c \in \mathbb{R}^{n}$, but, in contrast with the primal feasible set (where $F_{s}=F$ is possible) we always have $\overline{\Lambda_{s}} \neq \Lambda$. In fact, defining $\lambda \in \mathbb{R}_{+}^{(T)}$ such that

$$
\lambda_{t}= \begin{cases}1, & t=s, \\ 0, & t \in T \backslash\{s\},\end{cases}
$$

we have $\lambda \in \Lambda\left(a_{s}\right)$, so that $\Lambda\left(a_{s}\right) \neq \bar{\Gamma}$ for all $\Gamma \subset \mathbb{R}_{+}^{(T \backslash\{s\})}$. So, no constraint in $\sigma$ is superfluous relative to the dual feasible set.

Appealing to this notation, we say that $s$ is superfluous relative to the dual optimal set (DOS) if $\overline{\Lambda_{s}^{*}}=\Lambda^{*}$.

The superfluous constraints and variables are related with other types of unnecessary information. For instance, we say that $s \in T$ is nonessential if its corresponding constraint $a_{s}^{\prime} x \geq b_{s}$ is not binding at the set of extreme points of $F^{*}(c)$ for all $c \in \mathbb{R}^{n}$. On the other hand, a variable is said to be extraneous (strongly extraneous) in a standard LP problem

$$
\operatorname{Max} c^{\prime} x \quad \text { s.t. } \quad A x=b, x \geq 0_{n}
$$

where $A$ and $c$ are fixed and $b$ is the parameter, if this variable vanishes at some (all) optimal solution for any vector $b$ such that the above LP problem is solvable. Translating these concepts to our general context ( $T$ arbitrary), we say that $s \in T$ is extraneous (strongly extraneous) if for every $c \in \mathbb{R}^{n}$, either $\Lambda^{*}(c)=\emptyset$ or $s \notin \operatorname{supp} \lambda$ for some (all, respectively) $\lambda \in \Lambda^{*}(c)$.

The paper is mainly intended to provide tests for checking each of the eight superfluity phenomena in parametric linear optimization we have just defined. These geometric characterizations are given in Sections from 3 to 6. From them, we obtain in Section 7 a diagram (Figure 1) showing all the connections between all these phenomena. 
The next example shows that all these phenomena not only are possible but may occur simultaneously.

Example 1.1 Let $\sigma=\left\{x_{1} \geq 1 ; x_{1} \geq-1\right\}$ in $\mathbb{R}^{2}$. It is easy to see that $s=2$ is redundant, POS, PVS and nonessential. In order to show that it is also DOS, DVS and strongly extraneous we shall discuss the position of $c$ relative to the so-called first moment cone of $\sigma, M:=$ cone $\left\{a_{t}, t \in T\right\}$. If $c \notin M, D(c)$ and $D_{s}(c)$ are inconsistent, so that $\Lambda^{*}(c)=\Lambda_{s}^{*}(c)=\emptyset$ and $v^{D}(c)=v_{s}^{D}(c)=-\infty$. If $c \in M$, we can write $c=\mu(1,0)^{\prime}$ with $\mu \geq 0$. We have $\Lambda_{2}(c)=\{\mu\}$ and $\Lambda_{2}^{*}(c)=\{\mu\}$, with $v_{2}^{D}(c)=\mu$. On the other hand, $\Lambda(c)=\left\{\left(\lambda_{1}, \lambda_{2}\right)^{\prime} \in \mathbb{R}_{+}^{2} \mid \lambda_{1}+\lambda_{2}=\mu\right\}$ and $\Lambda^{*}(c)=\left\{(\mu, 0)^{\prime}\right\}$, with $v^{D}(c)=\mu$. Hence, $\overline{\Lambda_{2}^{*}(c)}=\Lambda^{*}(c)$ and $v_{2}^{D}(c)=v^{D}(c)$ for all $c \in \mathbb{R}^{2}$. Consequently, $s=2$ is DOS and DVS. Moreover, if $\Lambda^{*}(c) \neq \emptyset$, then it is a singleton set and the second coordinate of its unique element is zero. Thus, $s$ is strongly extraneous.

The first papers dealing with redundancy are due to Boot [4] and Charnes, Cooper and Thompson [5]. Since then many works have been written on this phenomenon (see, e.g., [10], [3], [7], and references therein). The extraneous variables were introduced in [5] and the strongly extraneous variables in [11] (see also [2], [6], and references therein). With the only exception of redundancy, fixing $c$ we get less restrictive concepts (i.e., excess of information phenomena in nonparametric linear optimization). For instance, Mauri [12] considered extraneous variables in LP whereas Goberna, Jornet and Molina [9] analyzed PVS and POS constraints in LP and LSIP.

Generally speaking, the existence of an excess of information in an optimization problem affects its theoretical properties and the computational efficiency of the numerical methods. Aardal [1] and Zhu and Broughan [13] have identified optimization problems in which the aggregation or the elimination of superfluous information provides important benefits. Concerning linear optimization, in LP the unfavorable effects of the excess of information outnumber the favorable ones ([11]), whereas the situation is the opposite in LSIP ([9]).

\section{Preliminaries}

Let us introduce the necessary notation and basic results (whose proofs can be found in $[8])$.

Given a set $\emptyset \neq X \subset \mathbb{R}^{n}$, we denote by cone $X$, span $X$, and conv $X$ the convex 
cone spanned by $X$, the linear span of $X$ and the convex hull of $X$, respectively. From the topological side, $\mathrm{cl} X$ denote the closure of $X$ and bd $X$ its boundary. If $X \neq \emptyset$ is a convex subset of a linear space, $\operatorname{extr} X$ and $O^{+} X$ denote its set of extreme points and its recession cone, respectively. By definition, $\operatorname{extr} \emptyset=\emptyset$.

We associate with $\sigma$ its first-moment cone, $M$, defined in Example 1.1 and its characteristic cone,

$$
K:=\text { cone }\left\{\left(\begin{array}{c}
a_{t} \\
b_{t}
\end{array}\right), t \in T ;\left(\begin{array}{c}
0_{n} \\
-1
\end{array}\right)\right\}
$$

$\sigma$ is consistent if and only if $\left(\begin{array}{c}0_{n} \\ 1\end{array}\right) \notin \mathrm{cl} K$. Moreover, $\sigma$ is strongly inconsistent (i.e., $\sigma$ contains at least a finite inconsistent subsystem) if and only if $\left(\begin{array}{c}0_{n} \\ 1\end{array}\right) \in K$.

We associate with $s \in T$ the characteristic and the first moment cone of $\sigma_{s}$, denoted by $K_{s}$ and $M_{s}$, and two intervals, $J_{s}$ and $I_{s}$, defined as

$$
J_{s}:=\left\{\alpha \in \mathbb{R} \mid\left(\begin{array}{c}
a_{s} \\
\alpha
\end{array}\right) \in K_{s}\right\} \subset I_{s}:=\left\{\alpha \in \mathbb{R} \mid\left(\begin{array}{c}
a_{s} \\
\alpha
\end{array}\right) \in K\right\},
$$

which can be empty, (open or closed) halflines or the whole real line $\mathbb{R}$. Obviously, $I_{s} \neq \emptyset$ whereas $J_{s} \neq \emptyset$ if and only if $a_{s} \in M_{s}$. Moreover, $\max \left\{b_{s}, \sup J_{s}\right\} \leq \sup I_{s} \leq+\infty$. It is easy to see that $\sup I_{s}=+\infty$ entails the inconsistency of $\sigma$ and, conversely, if $\sigma$ is strongly inconsistent, then $\sup I_{s}=+\infty$. Similarly, $\sup J_{s}=+\infty$ entails the inconsistency of $\sigma_{s}$ and, conversely, if $\sigma_{s}$ is strongly inconsistent and $a_{s} \in M_{s}$, then $\sup J_{s}=+\infty$.

Let $H_{s}:=\left\{x \in \mathbb{R}^{n} \mid a_{s}^{\prime} x=b_{s}\right\}$ (a hyperplane if $a_{s} \neq 0_{n}$ ). Then $\sigma_{s} \cup\left\{a_{s}^{\prime} x=b_{s}\right\}$ is a linear representation of $F \cap H_{s}$. If $\sigma$ is consistent and $s$ (or $a_{s}^{\prime} x \geq b_{s}$ ) is nonbinding (i.e., $F \cap H_{s}=\emptyset$ ), then $s$ is redundant. In particular, if $\sigma_{s} \cup\left\{a_{s}^{\prime} x=b_{s}\right\}$ is strongly inconsistent, then $s$ is called strongly redundant. If $\sigma$ is consistent, $s$ is strongly redundant if and only if there exists some $\varepsilon>0$ such that $\left(\begin{array}{c}a_{s} \\ b_{s}+\varepsilon\end{array}\right) \in K_{s}$.

Lemma 2.1 If $\sigma$ is not strongly inconsistent and $J_{s} \neq I_{s}$, then $\max I_{s}=b_{s}$.

Proof. We suppose that $\sigma$ is not strongly inconsistent and $I_{s} \backslash J_{s} \neq \emptyset$. If $\alpha \in J_{s}$ and $\beta \in I_{s} \backslash J_{s}$, then $\alpha<\beta$. 
Let $\beta \in I_{s} \backslash J_{s}$. Since $\left(\begin{array}{c}a_{s} \\ \beta\end{array}\right) \in K \backslash K_{s}$, we can write

$$
\left(\begin{array}{c}
a_{s} \\
\beta
\end{array}\right)=\left(\begin{array}{c}
a \\
b
\end{array}\right)+\delta\left(\begin{array}{c}
a_{s} \\
b_{s}
\end{array}\right)
$$

with $\left(\begin{array}{c}a \\ b\end{array}\right) \in K_{s}$ and $\delta>0$. Two cases can arise for $\delta$.

Case 1: $\delta<1$. From (1) we get

$$
\frac{1}{1-\delta}\left(\begin{array}{c}
a \\
b
\end{array}\right)=\frac{1}{1-\delta}\left(\begin{array}{c}
a_{s}(1-\delta) \\
\beta-\delta b_{s}
\end{array}\right)=\left(\begin{array}{c}
a_{s} \\
\frac{\beta-\delta b_{s}}{(1-\delta)}
\end{array}\right) \in K_{s} .
$$

Then, $\frac{\beta-\delta b_{s}}{(1-\delta)} \leq \sup J_{s}<b_{s}$. Thus, $\beta-\delta b_{s}<b_{s}-\delta b_{s}$, i.e., $\beta<b_{s}$.

Case 2: $\delta \geq 1$. Again from (1) we get

$$
\left(\begin{array}{c}
a_{s}(1-\delta) \\
\beta-\delta b_{s}
\end{array}\right)+(\delta-1)\left(\begin{array}{c}
a_{s} \\
b_{s}
\end{array}\right)=\left(\beta-b_{s}\right)\left(\begin{array}{c}
0_{n} \\
1
\end{array}\right) \in K
$$

and this entails, since $\sigma$ is not strongly inconsistent, that $\beta-b_{s} \leq 0$, i.e., $\beta \leq b_{s}$.

We have shown that $\beta \leq b_{s}$ for all $\beta \in I_{s} \backslash J_{s}$, so that $\sup I_{s} \leq b_{s}$. Since $b_{s} \in I_{s}$ we conclude that $\max I_{s}=b_{s}$.

Now assume that $\sigma$ is consistent. An inequality $a^{\prime} x \geq b$ is consequence of $\sigma$ if and only if $\left(\begin{array}{c}a \\ b\end{array}\right) \in \operatorname{cl} K$ (Farkas' Lemma). So, $K$ is closed if and only if every consequence of $\sigma$ is also the consequence of a finite subsystem. In this case $\sigma$ is said to be FarkasMinkowsy (FM). If $\sigma$ is an ordinary system (i.e., $|T|<\infty$ ), then it is FM.

With respect to the dual problem $D(c)$, we have

$$
v^{D}(c)=\sup \left\{\alpha \in \mathbb{R} \mid\left(\begin{array}{c}
c \\
\alpha
\end{array}\right) \in K\right\}
$$

(and this is different of $-\infty$ if and only if $c \in M$ ).

\section{Superfluous constraints relative to the primal mappings}

Proposition 3.1 Given $s \in T$, the following statements are equivalent to each other:

(i) $s$ is redundant.

(ii) $s$ is PVS. 
(iii) $s$ is POS.

(iv) $\left\{\left(\begin{array}{c}a_{s} \\ b_{s}\end{array}\right),\left(\begin{array}{c}0_{n} \\ 1\end{array}\right)\right\} \cap \mathrm{cl} K_{s} \neq \emptyset$.

Proof. If $a_{t}=0_{n}$ for all $t \in T$, it is easy to prove that the statements (i)-(iv) only fail (simultaneously) when $b_{t} \leq 0$ for all $t \in T \backslash\{s\}$ and $b_{s}>0$ (consider $c=0_{n}$ ). So we can assume that $\left\{a_{t}, t \in T\right\} \neq\left\{0_{n}\right\}$.

First we show that (i) $\Leftrightarrow($ ii $) \Leftrightarrow($ iii) discussing three possible cases.

Case 1: $\sigma$ and $\sigma_{s}$ are consistent.

(i) $\Rightarrow$ (iii) $\Rightarrow$ (ii) are trivial. In order to prove that (ii) $\Rightarrow$ (i) we assume that (i) fails. let $\bar{x} \in$ $F_{s} \backslash F$, i.e., $a_{t}^{\prime} \bar{x} \geq b_{t}$, for all $t \in T \backslash\{s\}$ and $a_{s}^{\prime} \bar{x}<b_{s}$. Then $v_{s}\left(a_{s}\right) \leq a_{s}^{\prime} \bar{x}<b_{s} \leq v\left(a_{s}\right)$, so that $v_{s} \neq v$, i.e., (ii) fails.

Case 2: $\sigma$ and $\sigma_{s}$ are inconsistent.

Since $F=F_{s}=\emptyset, F^{*}(c)=F_{s}^{*}(c)=\emptyset$ for all $c \in \mathbb{R}^{n}$ so that statements (i)-(iii) hold.

Case 3: $\sigma$ is inconsistent and $\sigma_{s}$ is consistent.

In this case $s$ is obviously nonredundant and we shall prove that (ii) and (iii) fail too. In fact, we have $F=\emptyset, F^{*}(c)=\emptyset$, and $v(c)=+\infty$ for all $c \in \mathbb{R}^{n}$. Moreover, the additional assumption guarantees that $\emptyset \neq F_{s} \neq \mathbb{R}^{n}$. Hence, there exists $\bar{x} \in \operatorname{bd} F_{s}$. Let $a^{\prime} x \geq b$ be a supporting halfspace for $F_{s}$ at $\bar{x}$. Then, $\bar{x} \in F_{s}^{*}(a)$. Since $F^{*}(a)=\emptyset \neq F_{s}^{*}(a)$ and $v_{s}(a)=b<+\infty=v(a)$, (ii) and (iii) fail.

Finally, let us observe that (i) holds if and only if either $F_{s}=\emptyset$ (in which case $F=\emptyset$ too) or $F_{s} \neq \emptyset$ and $a_{s}^{\prime} x \geq b_{s}$ for all $x \in F_{s}$, i.e., either $\left(\begin{array}{c}0_{n} \\ 1\end{array}\right) \in \operatorname{cl} K_{s}$ or $\left(\begin{array}{l}a_{s} \\ b_{s}\end{array}\right) \in \operatorname{cl} K_{s}$ (Farkas’ Lemma), i.e., (iv) holds.

If $s$ is superfluous relative to the primal mappings and $\sigma_{s}$ is consistent, then, from (iv), $a_{s} \in \operatorname{cl} M_{s}$. Nevertheless $a_{s} \in M_{s}$ could fail as the next example shows.

Example 3.1 Let $\sigma=\left\{(1-t) x_{1}+t x_{2} \geq 0, t \in[0,1]\right\}$ and $s=1$. It is easy to see that $F_{1}=F=\mathbb{R}_{+}^{2}$, so that $s=1$ is redundant. Nevertheless $a_{1}=(0,1)^{\prime} \notin M_{1}$.

\section{Superfluous variables relative to the dual value}

Proposition 4.1 Given $s \in T$, the following statements are equivalent to each other: (i) $s$ is DVS. 
(ii) $v_{s}^{D}\left(a_{s}\right)=v^{D}\left(a_{s}\right) \neq-\infty$.

(iii) $a_{s} \in M_{s}$ and $\sup J_{s}=\sup I_{s}$.

Proof. (i) $\Rightarrow$ (ii) From (i) we get $v_{s}^{D}\left(a_{s}\right)=v^{D}\left(a_{s}\right)$. Moreover, taking $\lambda \in \mathbb{R}_{+}^{(T)}$ such that $\lambda_{t}=1$ if $t=s$ and $\lambda_{t}=0$ otherwise, we have $\lambda \in \Lambda\left(a_{s}\right)$. Then $v^{D}\left(a_{s}\right) \geq \Psi(\lambda)=b_{s}>-\infty$.

(ii) $\Rightarrow$ (iii) Assume that $v_{s}^{D}\left(a_{s}\right)=v^{D}\left(a_{s}\right) \neq-\infty$. Since $D_{s}\left(a_{s}\right)$ is consistent, $a_{s} \in M_{s}$. Moreover, by (2),

$$
\sup J_{s}=v_{s}^{D}\left(a_{s}\right)=v^{D}\left(a_{s}\right)=\sup I_{s}
$$

(iii) $\Rightarrow$ (i) We assume that (iii) holds. Since $a_{s} \in M_{s}, M=M_{s}$.

Given $c \in \mathbb{R}^{n}$, if $c \notin M=M_{s}$, we have $v^{D}(c)=v_{s}^{D}(c)=-\infty$. Hence, we take $c \in M$. We have to prove that $v^{D}(c) \leq v_{s}^{D}(c)$, i.e., that $\alpha<v^{D}(c)$ entails $\alpha<v_{s}^{D}(c)$.

Given $\alpha<v^{D}(c)$, there exists $\lambda \in \mathbb{R}_{+}^{(T)}$ and $\beta>\alpha$ such that

$$
\sum_{t \in T} \lambda_{t}\left(\begin{array}{c}
a_{t} \\
b_{t}
\end{array}\right)=\sum_{t \in T \backslash\{s\}} \lambda_{t}\left(\begin{array}{c}
a_{t} \\
b_{t}
\end{array}\right)+\lambda_{s}\left(\begin{array}{c}
a_{s} \\
b_{s}
\end{array}\right)=\left(\begin{array}{c}
c \\
\beta
\end{array}\right) .
$$

If $\lambda_{s}=0$, we have $\alpha<\beta \leq v_{s}^{D}(c)$. So we assume $\lambda_{s}>0$.

Since $\left(\begin{array}{c}a_{s} \\ b_{s}\end{array}\right) \in K$, we have $b_{s} \leq \sup I_{s}=\sup J_{s}$.

Given $\varepsilon>0$, arbitrarily small, there exists $\gamma \in \mathbb{R}_{+}^{(T \backslash\{s\})}, \eta \geq 0$ and $\delta \in \mathbb{R}$ such that $b_{s}-\frac{\varepsilon}{\lambda_{s}}<\delta$ and

Then,

$$
\sum_{t \in T \backslash\{s\}} \gamma_{t}\left(\begin{array}{c}
a_{t} \\
b_{t}
\end{array}\right)+\left(\begin{array}{c}
0_{n} \\
-\eta
\end{array}\right)=\left(\begin{array}{c}
a_{s} \\
\delta
\end{array}\right)
$$

$$
\left(\begin{array}{c}
a_{s} \\
b_{s}
\end{array}\right)=\left(\begin{array}{c}
a_{s} \\
\delta
\end{array}\right)+\left(\begin{array}{c}
0_{n} \\
b_{s}-\delta
\end{array}\right)=\sum_{t \in T \backslash\{s\}} \gamma_{t}\left(\begin{array}{c}
a_{t} \\
b_{t}
\end{array}\right)+\left(\begin{array}{c}
0_{n} \\
b_{s}-\delta-\eta
\end{array}\right) .
$$

Combining (3) and (4) we obtain

$$
\left(\begin{array}{c}
c \\
\beta+\lambda_{s}\left(\delta-b_{s}+\eta\right)
\end{array}\right)=\sum_{t \in T \backslash\{s\}}\left(\lambda_{t}+\lambda_{s} \gamma_{t}\right)\left(\begin{array}{c}
a_{t} \\
b_{t}
\end{array}\right) \in K_{s},
$$

so that

$$
v_{s}^{D}(c) \geq \beta+\lambda_{s}\left(\delta-b_{s}+\eta\right)>\beta-\varepsilon>\beta>\alpha .
$$


This completes the proof.

In particular, if $K_{s}=K$ (e.g., if $a_{s}^{\prime} x \geq b_{s}$ is repeated), then $s$ is DVS.

Corollary 4.1 If $s$ is DVS, then $a_{s} \in M_{s}$. The converse statement holds if $\sigma_{s}$ is strongly inconsistent.

Proof. The direct statement is straightforward consequence of Proposition 4.1.

Now we assume that $a_{s} \in M_{s}$ and $\sigma_{s}$ is strongly inconsistent. Take an arbitrary $\alpha \in J_{s}$. Then for all $\delta \geq 0$ we have

$$
\left(\begin{array}{c}
a_{s} \\
\alpha+\delta
\end{array}\right)=\left(\begin{array}{c}
a_{s} \\
\alpha
\end{array}\right)+\delta\left(\begin{array}{c}
0_{n} \\
1
\end{array}\right) \in K_{s}+\text { cone }\left\{\left(\begin{array}{c}
0_{n} \\
1
\end{array}\right)\right\}=K_{s}
$$

so that $\alpha+\delta \in J_{s}$. Hence $+\infty=\sup J_{s} \leq \sup I_{s}$ and Proposition 4.1 applies again.

Proposition 4.2 If $s$ is DVS, then $s$ is redundant.

Proof. Assume that $s$ is nonredundant. Then, according to Proposition 3.1, we have $\left(\begin{array}{c}a_{s} \\ b_{s}\end{array}\right) \notin \operatorname{cl} K_{s}$. We shall prove that $\beta:=\sup J_{s}<b_{s}$. In fact, if $\beta \geq b_{s}$, then there exists a nondecreasing sequence of scalars, $\left\{\beta_{r}\right\}_{r \in \mathbb{N}}$, such that $\left(\begin{array}{c}a_{s} \\ \beta_{r}\end{array}\right) \in K_{s}$ for all $r \in \mathbb{N}$ and $\lim _{r} \beta_{r}=\beta \geq b_{s}$. Then,

$$
\left(\begin{array}{c}
a_{s} \\
b_{s}
\end{array}\right)=\lim _{r}\left(\begin{array}{c}
a_{s} \\
\beta_{r}
\end{array}\right)+\left(\begin{array}{c}
0_{n} \\
b_{s}-\beta
\end{array}\right) \in \operatorname{cl} K_{s}
$$

in contradiction with the assumption. Hence, by (2), $v_{s}^{D}\left(a_{s}\right)=\beta<b_{s} \leq v^{D}\left(a_{s}\right)$, and $s$ cannot be DVS.

The converse statement of Proposition 4.2 is not true, as Example 3.1 shows (recall that $\left.a_{1} \notin M_{1}\right)$.

\section{Extraneous variables and nonessential constraints}

Proposition 5.1 Given $s \in T$, the following statements are equivalent to each other:

(i) $s$ is extraneous.

(ii) If $D\left(a_{s}\right)$ is solvable, then $D_{s}\left(a_{s}\right)$ is solvable and $v_{s}^{D}\left(a_{s}\right)=v^{D}\left(a_{s}\right)$.

(iii) If there exists $\max I_{s} \in \mathbb{R}$, then $I_{s}=J_{s}$. 
Proof. (i) $\Rightarrow$ (ii) We assume that (i) holds and $D\left(a_{s}\right)$ is solvable.

Since $s$ is extraneous and $\Lambda^{*}\left(a_{s}\right) \neq \emptyset$, there exists $\lambda^{*} \in \Lambda^{*}\left(a_{s}\right)$ such that $s \notin \operatorname{supp} \lambda^{*}$. Then the restriction of $\lambda^{*}$ to $T \backslash\{s\}$ is a feasible solution of $D_{s}\left(a_{s}\right)$ such that the value of the objective functional is $\Psi\left(\lambda^{*}\right)=v^{D}\left(a_{s}\right) \geq v_{s}^{D}\left(a_{s}\right)$. Hence, $D_{s}\left(a_{s}\right)$ is solvable and $v_{s}^{D}\left(a_{s}\right)=v^{D}\left(a_{s}\right)$.

(ii) $\Rightarrow$ (iii) We assume that (ii) holds and $\bar{\alpha}=\max I_{s} \in \mathbb{R}$. Then, recalling (2), $D\left(a_{s}\right)$ is solvable and $v^{D}\left(a_{s}\right)=\bar{\alpha}$. Moreover, $\sup J_{s}=v_{s}^{D}\left(a_{s}\right)=v^{D}\left(a_{s}\right)=\bar{\alpha}$ and, due to the solvability of $D_{s}\left(a_{s}\right)$, we have $\max I_{s}=\bar{\alpha}=\max J_{s}$ and so $\left.\left.I_{s}=J_{s}=\right]-\infty, \bar{\alpha}\right]$.

(iii) $\Rightarrow$ (i) We assume (iii). Let $c \in M$ such that $\Lambda^{*}(c) \neq \emptyset$. Then, there exists $\gamma \in \mathbb{R}_{+}^{(T)}$ such that

$$
c=\sum_{t \in T} \gamma_{t} a_{t} \text { and } v^{D}(c)=\sum_{t \in T} \gamma_{t} b_{t}
$$

If $\gamma_{s}=0$ we have finished. So, we assume that $\gamma_{s}>0$. We shall obtain another optimal solution of $D(c)$ which vanishes at $s$.

First we prove that $v^{D}\left(a_{s}\right)=b_{s}$. Since $v^{D}\left(a_{s}\right) \geq b_{s}$ we shall assume that $v^{D}\left(a_{s}\right)>b_{s}$ and we shall get a contradiction. Let $\mu \in \mathbb{R}_{+}^{(T)}$ such that $\sum_{t \in T} \mu_{t} a_{t}=a_{s}$ and $b_{s}<\sum_{t \in T} \mu_{t} b_{t}$. Defining $\lambda \in \mathbb{R}_{+}^{(T)}$ as

$$
\lambda_{t}:= \begin{cases}\gamma_{t}+\gamma_{s} \mu_{t}, & \text { if } t \neq s \\ \gamma_{s} \mu_{s}, & \text { if } t=s\end{cases}
$$

we have

so that $\lambda \in \Lambda(c)$. Similarly,

$$
\begin{gathered}
\sum_{t \in T} \lambda_{t} a_{t}=\sum_{t \in T \backslash\{s\}}\left(\gamma_{t}+\gamma_{s} \mu_{t}\right) a_{t}+\gamma_{s} \mu_{s} a_{s} \\
=\sum_{t \in T \backslash\{s\}} \gamma_{t} a_{t}+\gamma_{s} \sum_{t \in T} \mu_{t} a_{t}=\sum_{t \in T \backslash\{s\}} \gamma_{t} a_{t}+\gamma_{s} a_{s}=c,
\end{gathered}
$$

$$
\begin{gathered}
\Psi(\lambda)=\sum_{t \in T} \lambda_{t} b_{t}=\sum_{t \in T \backslash\{s\}}\left(\gamma_{t}+\gamma_{s} \mu_{t}\right) b_{t}+\gamma_{s} \mu_{s} b_{s} \\
=\sum_{t \in T \backslash\{s\}} \gamma_{t} b_{t}+\gamma_{s} \sum_{t \in T} \mu_{t} b_{t}>\sum_{t \in T \backslash\{s\}} \gamma_{t} b_{t}+\gamma_{s} b_{s}=v^{D}(c),
\end{gathered}
$$

and this is a contradiction.

By (2), we have $b_{s}=v^{D}\left(a_{s}\right)=\max I_{s}$ and, recalling (iii), we get $b_{s} \in I_{s}=J_{s}$. Thus 
there exists $\rho \in \mathbb{R}_{+}^{(T \backslash\{s\})}$ and $\xi \geq 0$ such that

$$
\left(\begin{array}{c}
a_{s} \\
b_{s}
\end{array}\right)=\sum_{t \in T \backslash\{s\}} \rho_{t}\left(\begin{array}{c}
a_{t} \\
b_{t}
\end{array}\right)+\xi\left(\begin{array}{c}
0_{n} \\
-1
\end{array}\right) .
$$

Finally we shall prove that $\eta \in \mathbb{R}_{+}^{(T)}$, defined as

$$
\eta_{t}:= \begin{cases}\gamma_{t}+\gamma_{s} \rho_{t}, & \text { if } t \neq s \\ 0, & \text { if } t=s\end{cases}
$$

satisfies $\eta \in \Lambda^{*}(c)$ (observe that $s \notin \operatorname{supp} \eta$ ). In fact,

$$
\begin{gathered}
\sum_{t \in T} \eta_{t} a_{t}=\sum_{t \in T \backslash\{s\}}\left(\gamma_{t}+\gamma_{s} \rho_{t}\right) a_{t} \\
=\sum_{t \in T \backslash\{s\}} \gamma_{t} a_{t}+\gamma_{s} \sum_{t \in T \backslash\{s\}} \rho_{t} a_{t}=\sum_{t \in T \backslash\{s\}} \gamma_{t} a_{t}+\gamma_{s} a_{s}=c,
\end{gathered}
$$

so that, $\eta \in \Lambda(c)$. On the other hand,

$$
\begin{gathered}
\Psi(\eta)=\sum_{t \in T} \eta_{t} b_{t}=\sum_{t \in T \backslash\{s\}}\left(\gamma_{t}+\gamma_{s} \rho_{t}\right) b_{t} \\
=\sum_{t \in T \backslash\{s\}} \gamma_{t} b_{t}+\gamma_{s} \sum_{t \in T \backslash\{s\}} \rho_{t} b_{t}=\Psi(\gamma)-\gamma_{s} b_{s}+\gamma_{s}\left(b_{s}+\xi\right) \geq v^{D}(c),
\end{gathered}
$$

so that $\eta \in \Lambda^{*}(c)$. The proof is complete.

Corollary 5.1 If $\left(\begin{array}{c}a_{s} \\ b_{s}\end{array}\right) \in K_{s}$, then $s$ is extraneous. This occurs if $s$ is redundant and $\sigma_{s}$ is FM.

Proof. If $\left(\begin{array}{c}a_{s} \\ b_{s}\end{array}\right) \in K_{s}$, then $K=K_{s}$ and $I_{s}=J_{s}$. Then statement (iii) in Proposition 5.1 trivially holds.

Now we assume that $s$ is redundant and $\sigma_{s}$ is FM. Then $a_{s}^{\prime} x \geq b_{s}$ is a consequence of $\sigma_{s}$ and Farkas' Lemma yields $\left(\begin{array}{c}a_{s} \\ b_{s}\end{array}\right) \in \operatorname{cl} K_{s}=K_{s}$.

Example 3.1 shows that the FM assumption cannot be removed in Corollary 5.1. In fact, $s=1$ is redundant (but it is not DVS: $v_{1}^{D}\left(a_{1}\right)=-\infty<0=v^{D}\left(a_{1}\right)$ ). Nevertheless, defining $\lambda^{*}$ such that $\lambda_{t}^{*}=1$ if $t=1$ and $\lambda_{t}^{*}=0$ otherwise, it is easy to see that $\Lambda\left(a_{1}\right)=\left\{\lambda^{*}\right\}$, so that $\Lambda^{*}\left(a_{1}\right)=\left\{\lambda^{*}\right\}$. Since $1 \in \operatorname{supp} \lambda^{*}, s=1$ is not extraneous.

Corollary 5.2 If $s$ is extraneous and $\sigma$ is not strongly inconsistent, then $\sup J_{s} \geq b_{s}$. 
Proof. We suppose that $\sup J_{s}<b_{s}$. Since $b_{s} \leq \sup I_{s}, I_{s} \backslash J_{s} \neq \emptyset$. By Lemma 2.1, we have $\max I_{s}=b_{s}$. Hence, $I_{s}=J_{s}$ by Proposition 5.1, in contradiction with $\sup J_{s}<b_{s}$.

Proposition 5.2 If $\sigma$ is strongly inconsistent then any $s \in T$ is strongly extraneous. Otherwise, $s$ is strongly extraneous if and only if $\sup J_{s}>b_{s}$. In the last case, if $\sigma$ is consistent, then $s$ is strongly redundant.

Proof. First we assume that $\sigma$ is strongly inconsistent, i.e., $\left(\begin{array}{c}0_{n} \\ 1\end{array}\right) \in K$. Let $s \in T$ arbitrary and let $\gamma \in \mathbb{R}_{+}^{(T)}$ such that

$$
\left(\begin{array}{c}
0_{n} \\
1
\end{array}\right)=\sum_{t \in T} \gamma_{t}\left(\begin{array}{c}
a_{t} \\
b_{t}
\end{array}\right)
$$

Then, if $\Lambda(c) \neq \emptyset$ (i.e., $c \in M), \gamma \in O^{+} \Lambda(c)$ and $\Psi(\gamma)=\sum_{t \in T} \gamma_{t} b_{t}=1$. In such case $D(c)$ is unbounded (and so $\Lambda^{*}(c)=\emptyset$ ) for every $c \in \mathbb{R}^{n}$ and $s$ turns out to be strongly extraneous.

Now we assume that $\sigma$ is not strongly inconsistent.

Suppose that $s$ is strongly extraneous. By Corollary 5.2, we know that $\sup J_{s} \geq b_{s}$. We shall assume that $\sup J_{s}=b_{s}$ and we shall obtain a contradiction. If $\sup I_{s}>b_{s}$, then $J_{s} \varsubsetneqq I_{s}$. Then, by Lemma 2.1, $\max I_{s}=b_{s}$ and so $v^{D}\left(a_{s}\right)=b_{s}$.

Consider $\lambda \in \mathbb{R}_{+}^{(T)}$ such that $\lambda_{t}=1$ if $t=s$ and $\lambda_{t}=0$ otherwise. It can be easily realized that $\lambda \in \Lambda\left(a_{s}\right)$ with $\Psi(\lambda)=b_{s}=v^{D}\left(a_{s}\right)$. Then $\lambda \in \Lambda^{*}\left(a_{s}\right)$ and $s \in \operatorname{supp} \lambda$, and this contradicts the assumption on $s$.

Conversely, if $\sup J_{s}>b_{s}$, there exists $\varepsilon>0$ such that $\left(\begin{array}{c}a_{s} \\ b_{s}+\varepsilon\end{array}\right) \in K_{s}$. Then we can write

$$
\left(\begin{array}{c}
a_{s} \\
b_{s}+\gamma
\end{array}\right)=\sum_{t \in T} \lambda_{t}^{1}\left(\begin{array}{c}
a_{t} \\
b_{t}
\end{array}\right)
$$

with $\lambda^{1} \in \mathbb{R}_{+}^{(T)}, \gamma \geq \varepsilon>0$ and $s \notin \operatorname{supp} \lambda^{1}$.

Let us suppose that $s$ is not strongly extraneous. Then, there exists $c \in \mathbb{R}^{n}$ and $\lambda^{2} \in \Lambda^{*}(c)$ such that $s \in \operatorname{supp} \lambda^{2}$. Hence,

$$
\left(\begin{array}{c}
c \\
v^{D}(c)
\end{array}\right)=\sum_{t \in T} \lambda_{t}^{2}\left(\begin{array}{c}
a_{t} \\
b_{t}
\end{array}\right) \text { with } \lambda_{s}^{2}>0 .
$$

Defining

$$
\lambda_{t}^{3}:= \begin{cases}\lambda_{t}^{2}+\lambda_{s}^{2} \lambda_{t}^{1}, & \text { if } t \neq s \\ 0, & \text { if } t=s\end{cases}
$$


we have

$$
\sum_{t \in T} \lambda_{t}^{3} a_{t}=\sum_{t \in T \backslash\{s\}} \lambda_{t}^{2} a_{t}+\lambda_{s}^{2} \sum_{t \in T \backslash\{s\}} \lambda_{t}^{1} a_{t}=c-\lambda_{s}^{2} a_{s}+\lambda_{s}^{2} a_{s}=c
$$

and

$$
\begin{gathered}
\sum_{t \in T} \lambda_{t}^{3} b_{t}=\sum_{t \in T \backslash\{s\}} \lambda_{t}^{2} b_{t}+\lambda_{s}^{2} \sum_{t \in T \backslash\{s\}} \lambda_{t}^{1} b_{t} \\
=v^{D}(c)-\lambda_{s}^{2} b_{s}+\lambda_{s}^{2}\left(b_{s}+\gamma\right)=v^{D}(c)+\lambda_{s}^{2} \gamma>v^{D}(c),
\end{gathered}
$$

in contradiction with $\lambda^{2} \in \Lambda^{*}(c)$. So, $s$ is strongly extraneous.

Finally, observe that $\sup J_{s}>b_{s}$ entails the existence of $\varepsilon>0$ such that $\left(\begin{array}{c}a_{s} \\ b_{s}+\varepsilon\end{array}\right) \in$ $K_{s}$. Since we are assuming that $\sigma$ is consistent, $s$ is strongly redundant.

Proposition 5.3 If $s$ is extraneous (strongly extraneous) and $\sigma$ is consistent ( $\sigma$ is not strongly inconsistent, respectively), then $s$ is DVS.

Proof. First we assume that $s$ is extraneous and $\sigma$ is consistent.

Since $a_{s} \in M_{s}$ (by Corollary 5.2), $-\infty<\sup I_{s}<+\infty$ and so $I_{s}$ is a halfline.

Two cases can arise:

Case 1: $I_{s}$ is closed. Then, by Proposition 5.1, $I_{s}=J_{s}$.

Case 2: $I_{s}$ is open. Let $\bar{\alpha}:=\sup I_{s}$ and $\bar{\beta}:=\sup J_{s}$. We shall prove that $\bar{\alpha}=\bar{\beta}$.

Since $b_{s} \in I_{s}$ and $I_{s}$ is open, $b_{s}<\bar{\alpha}$. Let $\varepsilon>0$ arbitrarily such that $b_{s}+\varepsilon<\bar{\alpha}$, i.e., $b_{s}+\varepsilon \in I_{s}$. Then we can write

$$
\left(\begin{array}{c}
a_{s} \\
b_{s}+\varepsilon
\end{array}\right)=\sum_{t \in T \backslash\{s\}} \lambda_{t}\left(\begin{array}{c}
a_{t} \\
b_{t}
\end{array}\right)+\lambda_{s}\left(\begin{array}{c}
a_{s} \\
b_{s}
\end{array}\right)+\mu\left(\begin{array}{c}
0_{n} \\
-1
\end{array}\right),
$$

with $\lambda \in \mathbb{R}_{+}^{(T)}$ and $\mu \geq 0$.

If $\lambda_{s} \geq 1$, then

$$
\left(\begin{array}{c}
0_{n} \\
\varepsilon
\end{array}\right)=\sum_{t \in T \backslash\{s\}} \lambda_{t}\left(\begin{array}{c}
a_{t} \\
b_{t}
\end{array}\right)+\left(\lambda_{s}-1\right)\left(\begin{array}{c}
a_{s} \\
b_{s}
\end{array}\right)+\mu\left(\begin{array}{c}
0_{n} \\
-1
\end{array}\right) \in K,
$$

contradicting the assumption on $\sigma$.

Alternatively, if $0 \leq \lambda_{s}<1$, from (5) we obtain

$$
\left(1-\lambda_{s}\right)\left(\begin{array}{c}
a_{s} \\
b_{s}+\varepsilon
\end{array}\right)=\sum_{t \in T \backslash\{s\}} \lambda_{t}\left(\begin{array}{c}
a_{t} \\
b_{t}
\end{array}\right)+\left(\begin{array}{c}
0_{n} \\
-\mu-\lambda_{s} \varepsilon
\end{array}\right) \in K_{s}
$$


so that, $\left(\begin{array}{c}a_{s} \\ b_{s}+\varepsilon\end{array}\right) \in K_{s}$ and $b_{s}+\varepsilon \leq \bar{\beta}$. Hence, $\bar{\alpha}=\bar{\beta}$.

Since $\sup I_{s}=\sup J_{s}$ in both cases, $s$ is DVS according to Proposition 4.1.

Now we suppose that $s$ is strongly extraneous and $\sigma$ is not strongly inconsistent.

By Proposition 5.2, $\sup J_{s}>b_{s}$. Moreover, since $\sigma$ is not strongly inconsistent, $I_{s}$ and $J_{s}$ can not be lines and reasoning as in the first part of the proof, we conclude that $s$ is DVS.

Proposition 5.4 If $\sigma$ is inconsistent then any $s \in T$ is nonessential. Otherwise, $s$ is nonessential if and only if $(\operatorname{extr} F) \cap H_{s}=\emptyset$.

Proof. The first statement is trivial. So we assume that $\sigma$ is consistent.

Assume that $(\operatorname{extr} F) \cap H_{s} \neq \emptyset$. Let $\bar{x} \in(\operatorname{extr} F) \cap H_{s}$. Then $\bar{x} \in\left(\operatorname{extr} F^{*}\left(a_{s}\right)\right) \cap H_{s}$ and so $s$ is essential.

Conversely assume that $s$ is essential. Let $c \in \mathbb{R}^{n}$ such that $\left(\operatorname{extr} F^{*}(c)\right) \cap H_{s} \neq \emptyset$. If $c=0_{n}$, then $F^{*}(c)=F$ and $(\operatorname{extr} F) \cap H_{s} \neq \emptyset$. So we can assume that $c \neq 0_{n}$.

Let $\bar{x} \in\left(\operatorname{extr} F^{*}(c)\right) \cap H_{s}$. Since $H:=\left\{x \in \mathbb{R}^{n} \mid c^{\prime} x=c^{\prime} \bar{x}\right\}$ is a supporting hyperplane to $F$ at $\bar{x}$, we have

$$
(\operatorname{extr} F) \cap H=\operatorname{extr}(F \cap H)=\operatorname{extr} F^{*}(c) .
$$

Hence $\bar{x} \in\left(\operatorname{extr} F^{*}(c)\right) \cap H_{s} \subset(\operatorname{extr} F) \cap H_{s}$ and $(\operatorname{extr} F) \cap H_{s} \neq \emptyset$.

Proposition 5.5 Let $\sigma$ be consistent and $s \in T$. Then the following statements hold:

(i) If $s$ is strongly redundant, then it is strongly extraneous.

(ii) If $s$ is strongly extraneous, then it is nonbinding.

(iii) If $s$ is nonbinding, then it is nonessential.

(iv) If $s$ is nonessential and extr $F \neq \emptyset$, then it is nonbinding.

Proof. (i) Since $\sigma_{s} \cup\left\{a_{s}^{\prime} x=b_{s}\right\}$ is strongly inconsistent there exist $\lambda \in \mathbb{R}_{+}^{(T \backslash\{s\})}, \alpha \in \mathbb{R}$ and $\mu \in \mathbb{R}_{+}$such that

$$
\left(\begin{array}{c}
0_{n} \\
1
\end{array}\right)=\sum_{t \in T \backslash\{s\}} \lambda_{t}\left(\begin{array}{c}
a_{t} \\
b_{t}
\end{array}\right)+\alpha\left(\begin{array}{c}
a_{s} \\
b_{s}
\end{array}\right)+\mu\left(\begin{array}{c}
0_{n} \\
-1
\end{array}\right) .
$$

If $\alpha \geq 0$, then $\left(\begin{array}{c}0_{n} \\ 1\end{array}\right) \in K$, contradicting the assumption on $\sigma$.

Since $\alpha<0$, multiplying by $|\alpha|^{-1}$ both members of (6), we get 


$$
\left(\begin{array}{c}
a_{s} \\
b_{s}+|\alpha|^{-1}(1+\mu)
\end{array}\right)=\sum_{t \in T \backslash\{s\}}|\alpha|^{-1} \lambda_{t}\left(\begin{array}{c}
a_{t} \\
b_{t}
\end{array}\right) \in K_{s}
$$

so that $\sup J_{s} \geq b_{s}+|\alpha|^{-1}(1+\mu)>b_{s}$ and the conclusion follows from Proposition 5.2. (ii) Again by Proposition 5.2, we have $\sup J_{s}>b_{s}$. Let $\varepsilon>0$ and $\lambda \in \mathbb{R}_{+}^{(T)}$ such that

$$
\left(\begin{array}{c}
a_{s} \\
b_{s}+\varepsilon
\end{array}\right)=\sum_{t \in T \backslash\{s\}} \lambda_{t}\left(\begin{array}{c}
a_{t} \\
b_{t}
\end{array}\right)+\lambda_{s}\left(\begin{array}{c}
0_{n} \\
-1
\end{array}\right) .
$$

Let $x \in F$. Multiplying by $\left(x^{\prime},-1\right)$ both members of (7), we get $a_{s}^{\prime} x-\left(b_{s}+\varepsilon\right) \geq 0$. Then $a_{s}^{\prime} x>b_{s}$ and so $x \notin H_{s}$. Hence $F \cap H_{s}=\emptyset$.

(iii) If $F \cap H_{s}=\emptyset$, then (extr $\left.F\right) \cap H_{s}=\emptyset$ and so $s$ is nonessential by Proposition 5.4.

(iv) We assume that extr $F \neq \emptyset$ and $s$ is binding.

Consider an arbitrary $\bar{x} \in F \cap H_{s}$. Since $F=($ conv extr $F)+0^{+} F$, there exist $p \in \mathbb{N}$, $\left\{x^{1}, \ldots, x^{p}\right\} \subset \operatorname{extr} F,\left\{\lambda_{1}, \ldots, \lambda_{p}\right\} \subset \mathbb{R}_{+}$, and $y \in 0^{+} F$ such that $\sum_{i=1}^{p} \lambda_{i}=1$ and $\bar{x}=\sum_{i=1}^{p} \lambda_{i} x^{i}+y$. Since $\bar{x} \in H_{s}$, we have

$$
b_{s}=a_{s}^{\prime} \bar{x}=\sum_{i=1}^{p} \lambda_{i} a_{s}^{\prime} x^{i}+a_{s}^{\prime} y \geq \sum_{i=1}^{p} \lambda_{i} b_{s}+0=b_{s},
$$

and this entails $a_{s}^{\prime} x^{i}=b_{s}$ for all $i \in\{1, \ldots, p\}$. Hence, $\left\{x^{1}, \ldots, x^{p}\right\} \subset(\operatorname{extr} F) \cap H_{s}$, so that $s$ is essential.

The assumption extr $F \neq \emptyset$ cannot be eliminated in (iv): replace -1 in the system of Example 1.1 with 1.

\section{Superfluous variables relative to the dual optimal set}

Example 6.1 Let $\sigma=\left\{x_{1} \geq 1 ; x_{1} \geq 1\right\}$ in $\mathbb{R}^{2}$ and $s=2$. Obviously $s$ is superfluous relative to the primal mappings, DVS (by Proposition 4.1) and extraneous (by Corollary 5.1). Nevertheless it is neither strongly extraneous nor DOS: observe that $\overline{\Lambda_{2}^{*}(c)} \neq \Lambda^{*}(c)$ for all $c \in M \backslash\left\{0_{2}\right\}=$ cone $\left\{(1,0)^{\prime}\right\} \backslash\left\{0_{2}\right\}$ since

$$
\Lambda_{2}^{*}(c)=\left\{\begin{array}{cl}
\{\mu\}, & \text { if } c=\mu(1,0)^{\prime}, \mu \geq 0, \\
\emptyset, & \text { if } c \notin M
\end{array}\right.
$$


and

$$
\Lambda^{*}(c)= \begin{cases}\operatorname{conv}\left\{(\mu, 0)^{\prime},(0, \mu)^{\prime}\right\}, & \text { if } c=\mu(1,0)^{\prime}, \mu \geq 0, \\ \emptyset, & \text { if } c \notin M .\end{cases}
$$

In fact, the last two concepts are basically equivalent, as the next result shows.

Proposition 6.1 Given $s \in T$ the following statements hold:

(i) If $\sigma$ is strongly inconsistent, then $s$ is DOS if and only if $D_{s}(c)$ is unsolvable for all $c \in \mathbb{R}^{n}$.

(ii) If $s$ is DOS, then $s$ is strongly extraneous, and the converse is true if $\sigma$ is not strongly inconsistent.

Proof. (i)We assume that $\sigma$ is strongly inconsistent. In such a case $\Lambda^{*}(c)=\emptyset$ for all $c \in \mathbb{R}^{n}$, so that $s$ is DOS if and only if $\Lambda_{s}^{*}(c)=\emptyset$ for all $c \in \mathbb{R}^{n}$.

(ii) Suppose that $s$ is DOS. If $\Lambda^{*}(c) \neq \emptyset$, taking $\lambda \in \Lambda^{*}(c)=\overline{\Lambda_{s}^{*}(c)}$ we have $\lambda_{s}=0$, i.e., $s \notin \operatorname{supp} \lambda$. Hence, $s$ is strongly extraneous.

Finally we suppose that $s$ is strongly extraneous and $\sigma$ is not strongly inconsistent. By Proposition 5.3, $s$ is DVS and by Proposition 4.1, $a_{s} \in M_{s}$ (i.e., $M=M_{s}$ ) and $\sup J_{s}=\sup I_{s}$ (i.e., $v_{s}^{D}(c)=v^{D}(c)$ ). Let us show that $\overline{\Lambda_{s}^{*}(c)}=\Lambda^{*}(c)$ for every $c \in \mathbb{R}^{n}$. If $c \notin M=M_{s}, \overline{\Lambda_{s}^{*}(c)}=\Lambda^{*}(c)=\emptyset$. Otherwise, $v^{D}(c)=v_{s}^{D}(c)=\sup J_{s}>b_{s}$ by Proposition 5.2 and two cases can arise:

Case 1: $v^{D}(c)=v_{s}^{D}(c)=+\infty$ and so $\overline{\Lambda_{s}^{*}(c)}=\Lambda^{*}(c)=\emptyset$.

Case 2: $b_{s}<v^{D}(c)=v_{s}^{D}(c)<+\infty$. If there exists $c \in M$ and $\lambda \in \Lambda^{*}(c)$ such that $\lambda \notin \overline{\Lambda_{s}^{*}(c)}$, then $\lambda_{s}>0$ and so $s \in \operatorname{supp} \lambda$, contradicting the assumption on $s$. Thus $\Lambda^{*}(c) \subset \overline{\Lambda_{s}^{*}(c)}$ whereas the opposite inclusion trivially holds in this case.

Hence, $\overline{\Lambda_{s}^{*}(c)}=\Lambda^{*}(c)$ for every $c \in \mathbb{R}^{n}$ and $s$ is DOS.

The next example shows that the equivalence in (ii) fails if $\sigma$ is strongly inconsistent. It also shows that we can have $\Lambda^{*}(c)=\overline{\Lambda_{s}^{*}(c)}$, for all $c \in \mathbb{R}^{n} \backslash\left\{0_{n}\right\}$, and nevertheless $\Lambda^{*}\left(0_{n}\right) \neq \overline{\Lambda_{s}^{*}\left(0_{n}\right)}$.

Example 6.2 Let us consider $\sigma=\left\{x_{2} \geq t, t \in\right] 0,1\left[;-x_{2} \geq 0\right\}$ in $\mathbb{R}^{2}$ and let us associate to the constraint $-x_{2} \geq 0$ the index $s=1$. It can be realized that $\sigma$ is strongly inconsistent (and so, all constraints are strongly extraneous and inessential), whereas $\Lambda^{*}(c)=\overline{\Lambda_{1}^{*}(c)}=\emptyset$, for all $c \in \mathbb{R}^{n} \backslash\left\{0_{2}\right\}, \Lambda^{*}\left(0_{2}\right)=\emptyset$ and $\Lambda_{1}^{*}\left(0_{2}\right)$ is a singleton set formed by the null function. Thus $s=1$ is not DOS (in fact, it is not superfluous in any sense). 


\section{Conclusion}

We have characterized in a geometric way all the phenomena of excess information in parametric linear optimization introduced in Section 1, so that most of these properties can be checked in practice (observe that $\sup J_{s}$ and $\sup I_{s}$ can be computed by solving suitable LP or LSIP problems). Moreover, these characterizations allowed us to prove the relationships summarized in Figure 1. Examples can be given showing that any existing relationship between these phenomena can be derived from this diagram.

If $|T|<\infty$ and $\sigma$ is consistent, then there exist two clusters of equivalent properties:

(A) redundant, PVS, POS, DVS, and extraneous.

(B) strongly redundant, DOS, strongly extraneous, nonbinding, and nonessential (provided $F$ does not contain lines). 


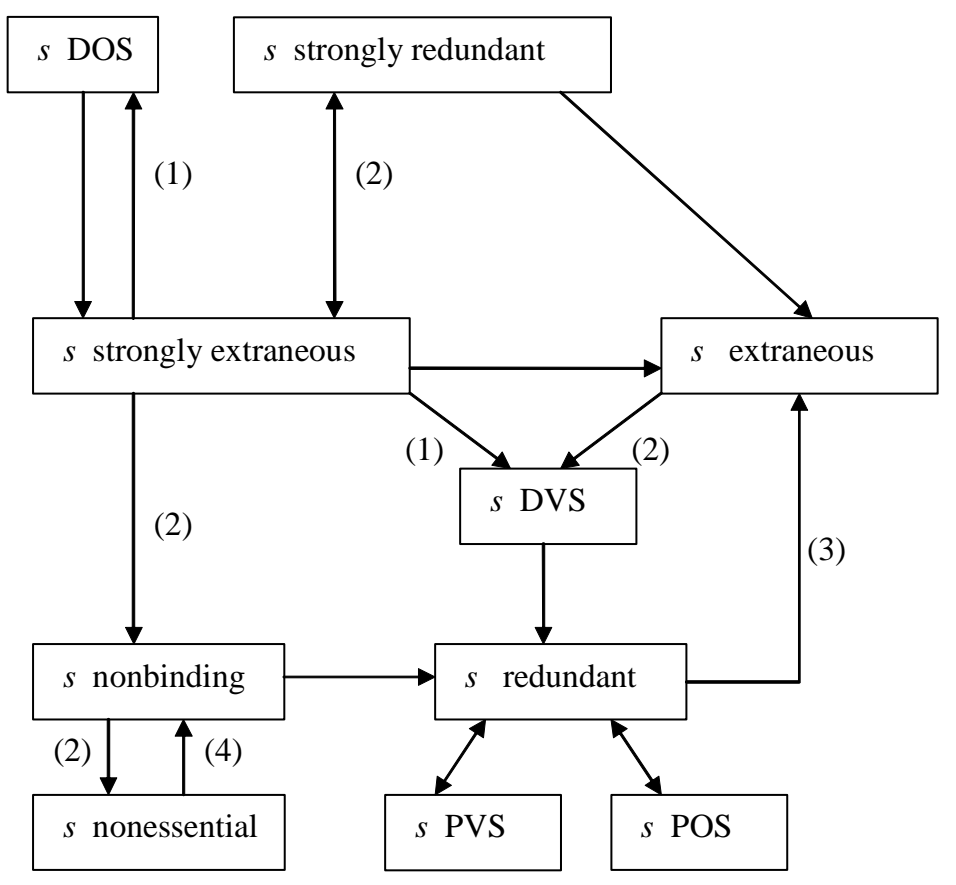

(1) If $\sigma$ is not strongly inconsistent.

(2) If $\sigma$ is consistent.

(3) If $\sigma_{\mathrm{s}}$ is FM (e.g., $|T|<\infty$ ).

(4) If $F \neq \varnothing$ does not contain lines.

Figure 1 


\section{References}

[1] Aardal, K., 1998, Reformulation of capacitated facility location problems: How redundant information can help. Annals of Operations Research, 82, 289-308.

[2] Boneh, A., Boneh, S., and Caron, R.J., 1993, Constraint Classification in Mathematical Programming. Mathematical Programming, 61, 61-73.

[3] Boneh, A., Boneh, S., and Caron, R.J., 1997, Redundancy. In: T. Gal et al. (Eds.), Advances in sensitivity analysis and parametric programming. Int. Ser. Oper. Res. Manag. Sci. Vol. 6, Dordrecht: Kluwer, 1-41.

[4] Boot, J.C.G., 1962, On Trivial and Binding Constraints in Programming Problems. Management Science, 8, 419-441.

[5] Charnes, A., Cooper, W.W., and Thompson, G.L., 1962, Some properties of redundant constraints and extraneous variables in direct and dual linear programming problems. Operations Research, 10, 711-723.

[6] Dulà, J.H., 1994, Geometry of Optimal Value Functions with Applications to Redundancy in Linear Programming. Journal of Optimization Theory and Applications, 81(1), 35-52.

[7] Goberna, M. A., Mira, J. A., and Torregrosa, G., 1998, Redundancy in Linear Inequality Systems. Numerical Functional Analysis and Optimization, 19(5-6), 529-548.

[8] Goberna, M.A. and López, M.A., 1998, Linear Semi-Infinite Optimization. (Chichester: Wiley).

[9] Goberna, M.A., Jornet, V., and Molina, M., 2003, Saturation in Linear Optimization. Journal of Optimization Theory and Applications, 117(2), 327-348.

[10] Greenberg, H.J., 1996, Consistency, redundancy, and implied equalities in linear systems. Annals of Mathematics and Artificial Intelligence, 17(1-2), 37-83.

[11] Karwan, M., Lofti, V., Telgen, J., and Zionts, S., 1983, Redundancy in Mathematical Programming. (Berlin: Springer).

[12] Mauri, M., 1975, Vincoli Superflui e Variabili Estranea in Programazioni Lineare. Ricerca Operativa 5, 21-42.

[13] Zhu, N. and Broughan, K., 1997, A Note on Reducing the Number of Variables in Integer 
Programming Problems. Computational Optimization and Applications, 8(3), 263-272. 